\title{
A NOTE ON HELSON'S EXISTENCE THEOREM
}

\author{
JUN-ICHI TANAKA
}

\begin{abstract}
In the almost periodic context, Helson showed that there exists a unitary function in every simply invariant subspace. We provide a short proof of this result in more general setting and give some applications.
\end{abstract}

Let $A$ be a weak-* Dirichlet algebra on probability measure space $(X, \varphi, \sigma)$. For $1 \leqslant p<\infty$, we define the space $H^{p}(\sigma)$ to be $[A]_{p}$, the $L^{p}(\sigma)$-closure of $A$, while $H^{\infty}(\sigma)$ is defined to be $[A]_{\infty}$, the weak-* closure of $A$ in $L^{\infty}(\sigma)$. A closed subspace $M$ in $L^{p}(\sigma), 1 \leqslant p<\infty$, (a weak-* closed subspace $M$ for $p=\infty)$ is said to be invariant if $A \cdot M \subset M$. An invariant subspace $M$ is called doubly invariant if $\bar{A} \cdot M \subset M$. We first prove the following:

THEOREM 1. Let $f$ be a nonnull function in $L^{p}(\sigma), 1 \leqslant p<\infty$, and let $E_{f}$ be the support set of $f$. Then there exists a $g$ in $[A \cdot f]_{p}$ such that $|g(x)|=1$ for o-a.e. $x$ in $E_{f}$.

In order to prove Theorem 1, we shall use the following well-known result which is a corollary of Szegö's theorem (see [4, Chapter 1], [9]).

LEMMA. If $f$ is a function in $L^{p}(\sigma), 1 \leqslant p \leqslant \infty$, such that $\log |f|$ is summable, then $f=p h$ with $p$ unitary and $h$ outer. The factoring is unique, up to multiplication of $q$ and $h$ by constant factors of modulus one.

Proof of Theorem 1. If $u=\min \left(1,|f|^{-1}\right)$, then $u$ is in $L^{\infty}(\sigma)$ and $\log u$ is summable. So there is an outer function $h$ in $H^{\infty}(\sigma)$ such that $|h|=u$ by the lemma. Since $[A \cdot f]_{p}=[A \cdot h f]_{p}$ and $\|h f\|_{\infty} \leqslant 1$, we may assume that $\|f\|_{\infty}$ $=1$. Next, we put

$$
F(n)=\left\{x ;(n+1)^{-1}<|f(x)|<n^{-1}\right\}
$$

for $n=1,2, \ldots$ Then $\sigma\left(E_{f} \backslash \cup_{n=1}^{\infty} F(n)\right)=0$. We define

$$
u_{n}(x)= \begin{cases}|f(x)|^{-1} & \text { for } x \text { in } F(n), \\ 2^{-(n+1)} & \text { for } x \text { in } X \backslash F(n) .\end{cases}
$$

Since $2^{-(n+1)} \leqslant u_{n}<n+1 \sigma$-a.e., there exists an outer function $h_{n}$ in $H^{\infty}(\sigma)$ such that $u_{n}=\left|h_{n}\right|$. Let $1 \leqslant p<\infty$. It is easy to see that $\left\|h_{n} f\right\|_{p} \leqslant \sigma(F(n))$ $+2^{-(n+1)}$, so we have

Received by the editors August 26, 1977.

AMS (MOS) subject classifications (1970). Primary 46J10, 46J15, 30A98; Secondary 22D40.

Key words and phrases. Weak-* Dirichlet algebra, invariant subspace, outer function, distant future, ergodic measure. 


$$
\sum_{n=1}^{\infty}\left\|h_{n} f\right\|_{p} \leqslant \sigma\left(E_{f}\right)+2^{-1}
$$

Since $h_{n} f$ is in $[A \cdot f]_{p}(n=1,2, \ldots)$, it follows that $\hat{g}(x)=\sum_{n=1}^{\infty} h_{n} f(x)$ is in $[A \cdot f]_{p}$. On the other hand, we have

$$
\begin{aligned}
|\hat{g}(x)| & \geqslant\left|h_{m} f(x)\right|-\left|\sum_{n \neq m} h_{n} f(x)\right| \\
& >1-\sum_{n=1}^{\infty} 2^{-(n+1)} \\
& =2^{-1} \text { for } \sigma \text {-a.e. } x \text { in } F(m) .
\end{aligned}
$$

Since $|\hat{g}(x)|<3 / 2$ for $\sigma$-a.e. $x$ in $X$, we can find an outer function $k$ in $H^{\infty}(\sigma)$ such that $k=|\hat{g}|^{-1}$ on $E_{f}$. So $g=\hat{g} \cdot k$ has the desired property. The proof is complete for $1 \leqslant p<\infty$. However, since $[A \cdot f]_{\infty}=[A \cdot f]_{1} \cap L^{\infty}(\sigma)$ (see [4, Chapter $1, \S 6]$ ), the conclusion holds for $p=\infty$.

From Theorem 1, we have this improvement of a theorem in [3].

Corollary. Let $M$ be an invariant subspace in $L^{p}(\sigma), 1<p<\infty$. Suppose that there exists a function $g$ in $M$ such that the zero set of $g, Z(g)$, is o-measure zero. Then $M$ contains a unitary function.

We can extend one result in [3] to the case of weak-* Dirichlet algebras. The proof is heavily dependent on Muhly's work [5].

For any subset $S$ of $L^{1}(\sigma)$, we write:

$$
|S|=\{|f| ; f \text { is in } S\} \text {. }
$$

THEOREM 2. Let $1 \leqslant p \leqslant \infty$. The following properties are equivalent:

(1) $H^{\infty}(\sigma)$ is a maximal weak-* closed subalgebra of $L^{\infty}(\sigma)$.

(2) For any nondoubly invariant subspace $M$ in $L^{p}(\sigma)$,

$$
|M|=\left|H^{p}(\sigma)\right| \text {. }
$$

Proof. $(1) \Rightarrow(2)$. Let $q$ be the conjugate index of $p: 1 / p+1 / q=1$. We set

$$
\tilde{M}=\left\{f \in L^{q}(\sigma) ; f g \text { is in } H^{1}(\sigma) \text { for any } g \text { in } M\right\} .
$$

Note that $\tilde{M}$ is an invariant subspace in $L^{q}(\sigma)$. It is easy to see that $M$ is doubly invariant iff $M \cdot \tilde{M}=\{0\}$. Therefore there exist nonnull bounded functions $h$ in $M$ and $k$ in $\tilde{M}$ such that $h k$ is in $H^{\infty}(\sigma)$ (see [4, Chapter $\left.1, \S 6\right]$ ). Since $H^{\infty}(\sigma)$ is maximal, it follows that $Z(h)$ and $Z(k)$ are $\sigma$-null sets by [5, Theorem]. So $M$ and $\tilde{M}$ contain unitary functions $\varphi$ and $\psi$ respectively by the above corollary. Since $\varphi \cdot H^{p}(\sigma) \subset M$ and $\psi \cdot M \subset H^{1}(\sigma) \cap L^{p}(\sigma)$, we have $|M|=\left|H^{p}(\sigma)\right|$.

(2) $\Rightarrow(1)$. Suppose that $H^{\infty}(\sigma)$ is nonmaximal. Then there exists a nonnull function $f$ in $H^{\infty}(\sigma)$ such that $\sigma(Z(f))>0$ by [5, Theorem]. Let $M=[A \cdot f]_{p}$. Then $M$ is invariant and $M \subset H^{p}(\sigma)$. Let $\chi_{Z(f)}$ denote the characteristic function of $Z(f)$. We can choose an outer function $h$ such that $|h|=\chi_{Z(f)}+$ 
1. So $|M| \&\left|H^{p}(\sigma)\right|$. Since $M$ contains no nonconstant real-valued functions, $M$ is not doubly invariant.

Next, we consider the condition (1) in [3]. Let $X$ be a compact Hausdorff space upon which the real line $R$ acts continuously as a topological transformation group. For any $x$ in $X$ and $t$ in $R, x+t$ will denote the translation of $x$ by $t$. Let $C[t, \infty)$ be the set of all continuous complex-valued functions $f$ such that the spectrum of $f$, denoted by $\operatorname{Sp}(f)$, is contained in $[t, \infty)$. We write $\mathfrak{A}=C[0, \infty)$, i.e., $\mathfrak{A}$ is the algebra of all continuous analytic functions. We refer the reader to [1] for the basic facts about spectra. For any finite regular Borel measure $\mu$, the distant future in $L^{2}(\mu)$ is defined to be $\cap_{-\infty<t<\infty} M_{t}(\mu)$, where $M_{t}(\mu)$ is the $L^{2}(\mu)$-closure of $C[t, \infty)$.

The following theorem is motivated by Muhly's remarks stated in [7, §5].

THEOREM 3. If $\sigma$ is an ergodic representing measure for $\mathfrak{A}$, then $\mathfrak{A}$ is a weak-* Dirichlet algebra in $L^{\infty}(\sigma)$. And, for any nonnegative function $w$ in $L^{p}(\sigma)$, $1 \leqslant p \leqslant \infty$, the following properties are equivalent:

(1) $\log w(x+t)$ belongs to $L^{1}\left(1 /\left(1+t^{2}\right) d t\right)$ as a function of $t$ for $\sigma$-a.e. $x$ in $X$.

(2) There exists a nonnull function $g$ in $H^{p}(\sigma)$ such that $|g|=w$.

Proof. Suppose that $f$ is a function in $L^{1}(\sigma)$ which annihilates $\mathfrak{A}+\overline{\mathfrak{A}}$. From [1, Proposition $2^{\prime}$ ], we see that the spectrum of $f \sigma, \operatorname{Sp}(f \sigma)$, is contained in $\{0\}$. By [6, Proposition 2.2 and Lemma 3], $|f| \sigma$ is an invariant measure. Suppose $f \sigma \neq 0$. Then, since $|f| \sigma$ is ergodic, $\mathfrak{A}+\overline{\mathfrak{A}}$ is weak-* dense in $L^{\infty}(|f| \sigma)$ by [6, Theorem I]. However, the Radon-Nikodym derivative $d f \sigma / d|f| \sigma$ annihilates $\mathfrak{A}+\overline{\mathfrak{A}}$. This is a contradiction. Therefore $\mathfrak{A}$ is a weak-* Dirichlet algebra in $L^{\infty}(\sigma)$.

Now we will show the equivalence of (1) and (2). It may be assumed that $\sigma$ is not a point mass, so $\sigma$ is quasi-invariant (see [6, Theorem III]). Recall that $H^{\infty}(\sigma)$ is maximal by [6, Corollary 3.1]. Let $p=2$. Since $\sigma$ is quasi-invariant, it is easy to see that (1) holds iff the distant future of $L^{2}\left(w^{2} \sigma\right)$ is the zero subspace by [8, Theorem I]. On the other hand, $[\mathfrak{A} w]_{2}=L^{2}(\sigma)$ iff $M_{0}\left(w^{2} \sigma\right)=$ $L^{2}\left(w^{2} \sigma\right)$. So, since $\sigma$ is ergodic quasi-invariant and not a point mass, we see that $[\mathfrak{A} w]_{2}$ is nondoubly invariant iff (1) holds by [8, Corollary 5.2 and Lemma 6.2]. Since $H^{\infty}(\sigma)$ is maximal, it follows from Theorem 2 that $\left|[\mathfrak{A} w]_{2}\right|=\left|H^{2}(\sigma)\right|$ iff $[\mathfrak{A} w]_{2}$ is not a doubly invariant subspace. Therefore we have the equivalence of (1) and (2) for the case $p=2$. Let $1 \leqslant p \leqslant \infty$. Since

$$
H^{p}(\sigma)=H^{1}(\sigma) \cap L^{p}(\sigma),
$$

it suffices to consider the case $p=1$. If $w=|g|$ where $g$ is a nonnull function in $H^{1}(\sigma)$, then there is an outer function $h$ in $H^{\infty}(\sigma)$ such that $g h$ is in $H^{2}(\sigma)$. Since $|g h|$ has the property of (1) by the above case, it follows that (2) implies (1). The other direction is easy, so the proof is complete.

\section{REFERENCES}

1. F. Forelli, Analytic and quasi-invariant measures, Acta Math. 118 (1967), 33-59.

2. T. Gamelin, Uniform algebras, Prentice-Hall, Englewood Cliffs, N. J., 1969. 
3. H. Helson, Compact groups with ordered duals. IV, Bull. London Math. Soc. 5 (1973), 67-69.

4. __, Analyticity on compact abelian groups, Algebras in Analysis, Academic Press, New York, 1975, pp. 1-62.

5. P. Muhly, Maximal weak-* Dirichlet algebras, Proc. Amer. Math. Soc. 36 (1972), 515-518.

6. __ Function algebras and flows. I, Acta Sci. Math. (Szeged) 35 (1973), 111-121.

7. Function algebras and flows. III, Math. Z. 136 (1974), 253-260.

8. The distant future, Indiana Math. J. 24 (1974), 149-159.

9. T. Srinivasan and J. Wang, Weak*-Dirichlet algebras, Function Algebras (Tulane Univ., 1965), Scott, Foresman, Chicago, Ill., 1966, pp. 216-249.

Departnant of Mathematics, Tsuru University, Tsuru CtTy, Yamanashi, 402 Japan 